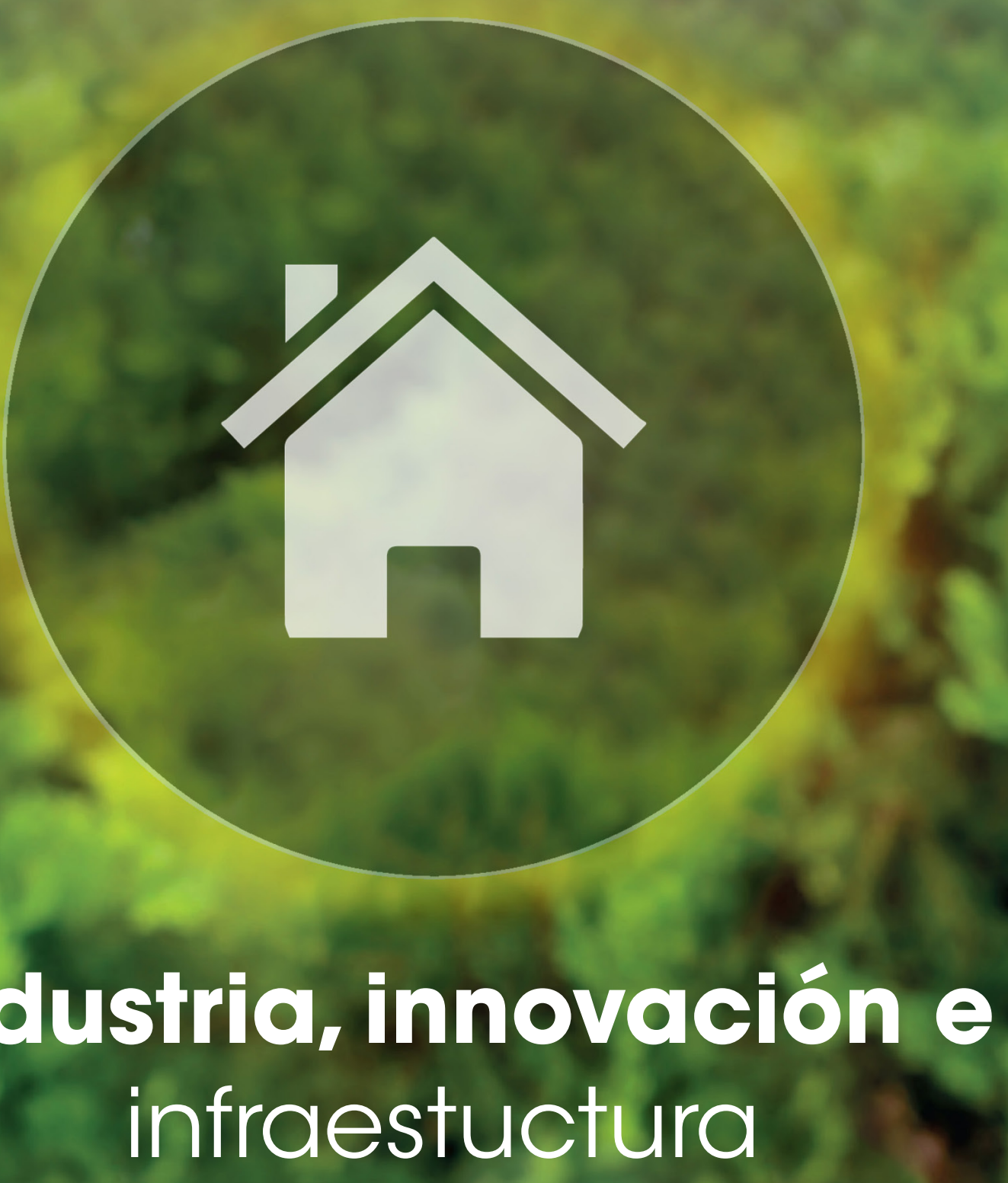




\section{ECONOMÍA CIRCULAR COMO ESTRATEGIA \\ PARA LA CONSTRUCCIÓN DE VÍAS EN \\ COLOMBIA. CASO DE ESTUDIO}

Sandra Liliana Uribe Celis ${ }^{10}$

Cristian Camilo Riveros Roa ${ }^{11}$

Luisa Julieth Rojas Porras ${ }^{12}$

Universidad de la Salle

\section{Resumen:}

El objetivo de la investigación es desarrollar una estrategia para la aplicación de la economía circular en la construcción de vías secundarias y terciarias, mediante la utilización de los residuos de construcción como materia prima en las capas granulares de las vías. Esto, según el panorama actual de la infraestructura vial en Colombia, la dificultad en la obtención de materiales para su construcción, el manejo inadecuado de los residuos de construcción y demolición y la implementación de la Estrategia Nacional de Economía Circular en Colombia en 2018. Para ello se analiza la factibilidad técnica a través de la revisión teórica del uso de los residuos en diferentes partes del mundo; el análisis de la factibilidad comercial, identificando la cantidad de material a reemplazar y el material disponible para su reutilización, y el análisis de la factibilidad económica, mediante la comparativa de presupuestos de la construcción de una vía con material convencional y una usando material reciclado, aplicado un estudio de caso en Sopó, Cundinamarca. Así, se determina la viabilidad del uso de los residuos de construcción para las carreteras y se diseña la estrategia para el aprovechamiento de los residuos y la reducción de materia prima.

\section{Palabras clave:}

economía circular, residuos de construcción, sostenibilidad, infraestructura vial.

10Docente investigadora de la Universidad de La Salle. Correo electrónico: sluribe@unisalle.edu.coInstituto "Estudiante del semillero SOSYMAT de la Universidad de La Salle. Correo electrónico: criveros44@unisalle.edu.co 12 Estudiante del semillero SOSYMAT de la Universidad de La Salle. Correo electrónico: Irojas76@unisalle.edu.co 


\begin{abstract}
:
The investigation objective is to develop a strategy to circular economy application at the secondary and tertiary routes construction through the use of construction waste as raw material at the granular layers of the roads. This, according to the current outlook of road infrastructure in Colombia, the difficulty obtaining materials for its construction, the inadequate management of construction and demolition waste and the National Strategy of Circular Economy implementation in Colombia in 2018. Thereby, it is analyze the technical feasibility through the theoretical review of the use of construction and demolition waste in different parts of the world; the analysis of the commercial feasibility, identifying the amount of material to replace and the material available for reuse and the analysis of the economic feasibility by comparing construction budgets of one way with conventional material and other one using recycled material, applied in a case of study in Sopó, Cundinamarca. Thereby, it is determined the viability of the construction waste use in road construction and it is designed a strategy waste utilization and raw material reduction.
\end{abstract}

\title{
Keywords:
}

circular economy, construction and demolition waste, sustainability, road infrastructure.

\section{Introducción}

En la construcción de edificaciones y carreteras se utilizan materiales extraídos de fuentes no renovables de la naturaleza; el uso de algunos de estos materiales de forma masiva genera impactos medioambientales y contaminantes [1]. Debido a los efectos que producen, en los últimos años se han implementado soluciones sostenibles en la industria de la construcción; buscan reducir las emisiones de dichas construcciones y generar un equilibrio 
utilizando los residuos generados por estas, mientras se realiza el ejercicio de la responsabilidad ambiental y social [2].

Por otra parte, en Colombia se está implementando el Acuerdo general para la terminación del conflicto y la construcción de la paz estable y duradera. Este propone el desarrollo de la infraestructura vial secundaria y terciaria como estrategia; busca la integración rural y el desarrollo socioeconómico de las zonas municipales y veredales apartadas debido al conflicto interno. El desarrollo de las redes secundarias y terciarias, que corresponden aproximadamente al 92\% de la red vial nacional, trabaja de la mano con la construcción sostenible en la búsqueda de materiales y nuevas alternativas que permitan la reducción de costos. Lo anterior garantiza la seguridad y funcionalidad de las vías del postconflicto [3].

En consecuencia, en el 2018 se realizó el lanzamiento de la Estrategia Nacional de Economía Circular con el fin de "fomentar la reducción, el reciclaje y la reutilización de los residuos y materiales" [4]; de allí que estén desarrollando alternativas con el uso de materiales reciclados. Según el Decreto 1115 de 2012 "Ios residuos generados por la construcción y la demolición representan recursos potenciales para la obtención de áridos reciclados, y también un sin número de materiales secundarios" [5]. Este tipo de productos reciclados cada vez tienen mayor presencia en el mercado; con su uso se pueden mitigar los efectos medioambientales asociados a la extracción en canteras y preservar así los recursos naturales [1].

Según lo anterior, en la presente investigación se evidencia el desarrollo de una metodología viable para implementar la economía circular en la construcción de carreteras secundarias y terciaras. Esto, a través del análisis de la factibilidad comercial y económica sobre la implementación de los Residuos de Construcción (RC) en la construcción de carreteras secundarias y terciarias. Además, parte de la viabilidad técnica del uso de dichos materiales en las construcciones viales. 


\section{Marco Teórico}

\section{Residuos de Construcción y Demolición (RCD).}

Los RCD son los residuos que se generan en el desarrollo de un proyecto constructivo, de recuperación o demolición. Este conjunto de residuos es considerado como residuo solido especial [6]; gracias a su tamaño, peso, volumen, composición, condiciones de almacenaje y transporte no puede ser manejado por el servicio público de aseo, es necesario acordar entre el usuario y una persona encargada de prestar dicho servicio especial [7]. Las categorías en las cuales se clasifican los RCD se encuentran detalladas en la siguiente tabla.

Tabla 1. Clasificación de los residuos de construcción y demolición.

\begin{tabular}{|l|l|l|l|}
\hline \multicolumn{1}{|c|}{ CATEGORÍA } & \multicolumn{1}{|c|}{ GRUPO } & \multicolumn{1}{c|}{ COASE } \\
\hline RCD Aprovechables & $\begin{array}{l}\text { I. Residuos comunes iner- } \\
\text { tes mezclados }\end{array}$ & Residuos pétreos & $\begin{array}{l}\text { Concretos, cerámicos, la- } \\
\text { drillos, arenas, gravas, can- } \\
\text { tos, bloques o fragmentos } \\
\text { de roca, baldosín, mortero } \\
\text { y materiales inertes que no } \\
\text { sobrepasen tamiz \#200 }\end{array}$ \\
\cline { 2 - 4 } & $\begin{array}{l}\text { I. Residuos comunes iner- } \\
\text { tes de material fino }\end{array}$ & $\begin{array}{l}\text { Residuos finos no expan- } \\
\text { sivos }\end{array}$ & $\begin{array}{l}\text { Arcillas, limos y residuos } \\
\text { inertes, poco o no plásticos } \\
\text { y expansivos que sobrepa- } \\
\text { sen el tamiz \#200 }\end{array}$ \\
\cline { 2 - 4 } & $\begin{array}{l}\text { Residuos finos expansivos } \\
\text { Arcillas y lodos inertes con } \\
\text { gran cantidad de finos al- } \\
\text { tamente expansivos que } \\
\text { sobrepasen el tamiz \#200 } \\
\text { plásticos }\end{array}$ \\
\hline
\end{tabular}




\begin{tabular}{|c|c|c|c|}
\hline & IV. Residuos metálicos & $\begin{array}{l}\text { Residuos de carácter me- } \\
\text { tálico }\end{array}$ & $\begin{array}{l}\text { Acero, hierro, cobre, alumi- } \\
\text { nio, estaño y zinc }\end{array}$ \\
\hline & \multirow[t]{2}{*}{ V. Residuos orgánicos } & Residuos de pendones & Residuos de tierra negra \\
\hline & & Residuos de cespedones & $\begin{array}{l}\text { Residuos vegetales y otras } \\
\text { especies bióticas }\end{array}$ \\
\hline \multirow[t]{2}{*}{ RCD No aprovechables } & \multirow[t]{2}{*}{$\begin{array}{l}\text { VI. Residuos contaminan- } \\
\text { tes }\end{array}$} & Residuos peligrosos & $\begin{array}{l}\text { Desechos de productos } \\
\text { químicos, emulsiones, al- } \\
\text { quitrán, pinturas, disol- } \\
\text { ventes orgánicos, aceites, } \\
\text { asfaltos, resinas, plastifi- } \\
\text { cantes, tintas, betunes, plo- } \\
\text { mo, desechos explosivos, } \\
\text { plastificantes, barnices, te- } \\
\text { jas de asbesto, entre otros } \\
\text { elementos peligrosos }\end{array}$ \\
\hline & & Residuos especiales & $\begin{array}{l}\text { Polietileno icopor, drywall, } \\
\text { lodos residuales de com- } \\
\text { puestos }\end{array}$ \\
\hline
\end{tabular}

Fuente: Guía para la elaboración del plan de gestión de Residuos de Construcción y Demolición (RCD) en obra, [8].

\section{Carreteras secundarias y terciarias.}

Una carretera es una infraestructura de transporte acondicionada dentro de una franja de terreno para permitir la circulación vehicular de manera continua, cómoda y segura [9]. Según el Manual de diseño geométrico de carreteras, las carreteras se clasifican según su funcionalidad y tipo de terreno. Para este caso se tiene en cuenta la clasificación según su funcionalidad primaria, secundaria o terciaria.

Las carreteras primarias son las troncales, transversales y accesos a capitales de Departamentos que integran las principales zonas de producción y consumo del país. Las carreteras secundarias son las vías que unen las cabeceras municipales entre sí y se conectan con una carretera primaria. Por último, las 
vías terciarias conectan las cabeceras municipales con veredas aledañas. Tanto las carreteras primarias como secundarias deben funcionar pavimentadas, en el caso de las vías terciarias también se puede considerar el uso de afirmado.

Una carretera está compuesta por una estructura principal, como principales elementos de soporte tiene la base, la subbase y la capa de rodadura. La subbase hace referencia a la capa granular localizada entre la subrasante y la base granular; el espesor de esta capa no debe ser inferior a 10 centímetros (cm) y superior a $20 \mathrm{~cm}$. Asimismo, la base granular debe cumplir con el mismo criterio en cuanto a espesor, la diferencia es la granulometría de la cual cada una de las capas está conformada. Estas capas:

[...] se construyen con materiales pétreos los cuales pueden provenir de la trituración de rocas y gravas, o podrán ser constituidos por una mezcla de productos de ambas procedencias (...) Las partículas de los agregados serán duras, resistentes y durables, sin excesos de partículas planas, alargadas, blandas o desintegrables y sin materia orgánica u otras sustancias perjudiciales [10].

Para la construcción de estas capas se deben tener en cuenta las normas del Instituto Nacional de Vías (INVIAS). Dichas normas especifican los requisitos del material a fondo, así como los ensayos a realizar para garantizar un óptimo funcionamiento y durabilidad de la vía.

\section{Estado actual de las vías en Colombia.}

El Plan Nacional de Desarrollo (2014-2018) habla sobre la construcción y mantenimiento de las vías, en especial las de tercer orden, como una estrategia para alcanzar los propósitos de integración territorial y asimismo aumentar el desarrollo económico, conectando los lugares más inasequibles con las principales urbes del país. Por otra parte, con la llegada del acuerdo de paz vienen una serie de planteamientos que proponen ayudar a la población vulnerada por el conflicto armado de nuestro país. 
Una de las posibles soluciones es el tema de la infraestructura vial; el Gobierno Nacional implementa un Plan Nacional de Vías terciarias que debe cumplir los siguientes criterios: la participación de la comunidad en la ejecución y seguimiento de las obras, el estímulo a la economía del sector (con la contratación a personal local como prioridad), la implementación de soluciones tecnológicas, la asistencia técnica por parte de la comunidad para garantizar la sostenibilidad de las obras y la sostenibilidad de las condiciones socioambientales.

Actualmente las vías secundarias y terciarias juegan un papel muy importante en la unificación nacional, regional y local. En total, estas vías abarcan un 92\% de la malla vial nacional, con 44.339 km (21\%) de red vial de segundo orden y 154.207 (71\%) de tercer orden o veredal [5].

\section{Economía circular en Colombia.}

Colombia es uno de los pioneros en la implementación de la economía circular. En noviembre del 2018 se realizó el lanzamiento de la Estrategia Nacional de la Economía Circular; iniciativa del Gobierno Nacional para promover las nuevas formas de producción, consumo y aprovechamiento de desechos. Como base de la economía circular, se definen nueve (9) R importantes en el desarrollo de esta estrategia:

- Repensar

- Reutilizar

- Reparar

- Restaurar

- Remanufacturar

- Reducir

- Re-proponer 
- Reciclar

- Recuperar

La implementación de la economía circular trae beneficios tanto ambientales como económicos. Por un lado, este término "se traduce en reducción de la extracción de materias primas, uso de fuentes de energía renovables, la reducción de residuos y emisiones, y la conservación y uso eficiente del agua" [6]. En cuanto al ámbito económico, se evidencia una reducción de costos en la materia prima mediante el aprovechamiento de recursos en repetidas ocasiones (reciclaje). La implementación de esta estrategia es un trabajo conjunto en el país de varios ministerios como el de Comercio, Vivienda, Educación, Transporte, Minas, Agricultura y Ambiente.

\section{Estimación de los residuos de construcción generados.}

La estimación de los residuos de construcción y demolición es una investigación que han desarrollado en diferentes países, en las últimas décadas. Diferentes autores han encontrado factores de generación que permiten estimar la cantidad de residuo generado en una construcción, según las condiciones constructivas locales. Por ejemplo, en al año 2014, realizaron una investigación en la ciudad de Villavicencio, Colombia; se analizan (cualitativa y cuantitativamente) los residuos generados por tres (3) construcciones de tipo residencial y tres (3) construcciones de tipo comercial para determinar un factor que permita evidenciar el volumen de residuo que se obtiene por cada metro cuadrado (m2) de construcción. Su resultado se expuso en el V Congreso de Ingeniería Civil de la Universidad Santo Tomás seccional Tunja.

La metodología ejecutada en la investigación consiste en escoger las construcciones de tipo residencial y comercial, recolectar los residuos de construcción de las obras que posteriormente fueron separados con ayuda de un tamiz 
de cinco milímetros (5mm). Como resultado surgen seis (6) grupos o tipos de materiales: agregados finos, agregados gruesos, concreto, mampostería, acero y otros. Además, cada tipo de material es pesado para determinar su porcentaje en los residuos, consiguiendo los siguientes resultados:

Figura 1. Composición de RC.

a)

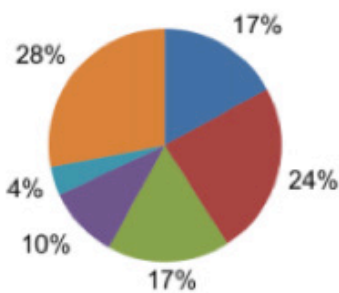

c)
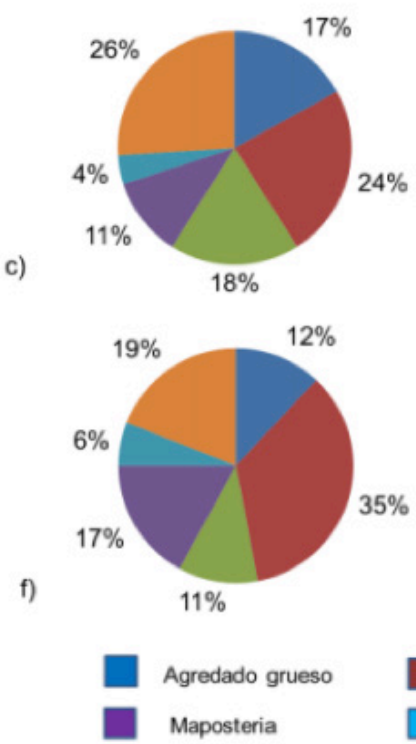

b)

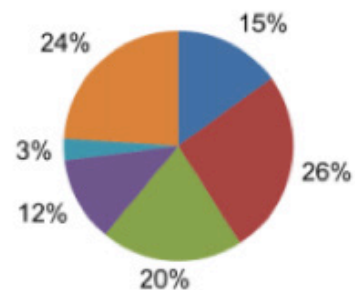

d)

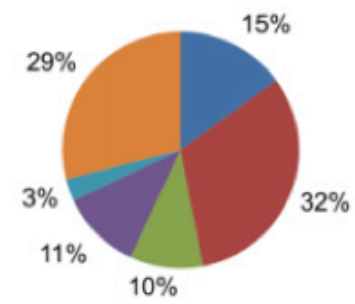

9

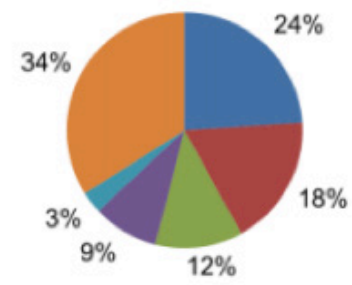

Agregado fino

Acero $\square$ Concreto

$\square$ Otros

Fuente: Estimación de generación y composición de residuos de construcción en la ciudad de Villavicencio, [11]. 
Tras obtener la cantidad de residuos de la obra en metros cúbicos (m3), proceden a relacionar dicho resultado con los m2 construidos; realizan el promedio de los factores obtenidos y determinan dicho factor (como el de generación de RC en la obra). Para la construcción comercial existe un factor de 0.08 m3/m2 y para la construcción residencial uno de 0.144 m3/m2. Dicho factor hace referencia al total de residuos generados, es decir, tanto el concreto como la madera, el acero y otros, sin embargo, en este caso no se usan todos estos materiales, únicamente los agregados finos, gruesos y el concreto.

En la Tabla 2 se presenta el resultado de los factores obtenidos por las construcciones seleccionadas en el estudio; a, b y c corresponden a las construcciones residenciales estudiadas y d, e y f corresponden a las construcciones comerciales seleccionadas respectivamente:

Tabla 2. Factor de generación RC

\begin{tabular}{|c|c|c|c|}
\hline ítem & Tipo & área $\left(\mathrm{m}^{2}\right)$ & Factor $\left(\mathrm{m}^{3} / \mathrm{m}^{2}\right)$ \\
\hline a & Vivienda & 198,44 & 0,151 \\
\hline b & Vivienda & 189,62 & 0,137 \\
\hline c & Vivienda & 186,67 & 0,144 \\
\hline d & Comercial & 420 & 0,086 \\
\hline e & Comercial & 1186,90 & 0,076 \\
\hline f & Comercial & 776,44 & 0,077 \\
\hline
\end{tabular}

Fuente: Estimación de generación y composición de residuos de construcción en la ciudad de Villavicencio, [11]. 
Los autores afirman que "la composición porcentual por residuos de concreto, bloques, arenas, gravas, ladrillo y tierra representan hasta un 50\% o más de los residuos" (Rodríguez Miranda \& Agudelo Varela, 2014).

Tabla 3. Porcentaje de material aprovechable.

\begin{tabular}{|l|c|c|c|}
\hline & a & b & c \\
\hline Agregado grueso & $17 \%$ & $17 \%$ & $15 \%$ \\
\hline Agregado fino & $24 \%$ & $24 \%$ & $26 \%$ \\
\hline Concreto & $17 \%$ & $18 \%$ & $20 \%$ \\
\hline SUMATORIA & $58 \%$ & $59 \%$ & $61 \%$ \\
\hline PROMEDIO & & & \\
\hline
\end{tabular}

Fuente: elaboración propia.

De acuerdo a la cantidad porcentual de cada uno de los materiales aprovechables en la construcción de carreteras (pétreos, agregados finos y gruesos, concreto, etc.), se determina el porcentaje de dichos materiales en las obras presentadas en el artículo y se realiza el promedio de los resultados obtenidos. El factor de generación de residuos de construcción corresponde al 59\%; permite determinar la cantidad de material a aprovechar de los residuos de construcción: 0.085m3/m2. 


\section{Metodología}

A continuación, se presenta el diagrama de la metodología utilizado para el desarrollo de la investigación.

Figura 2. Esquema de la metodología empleada.

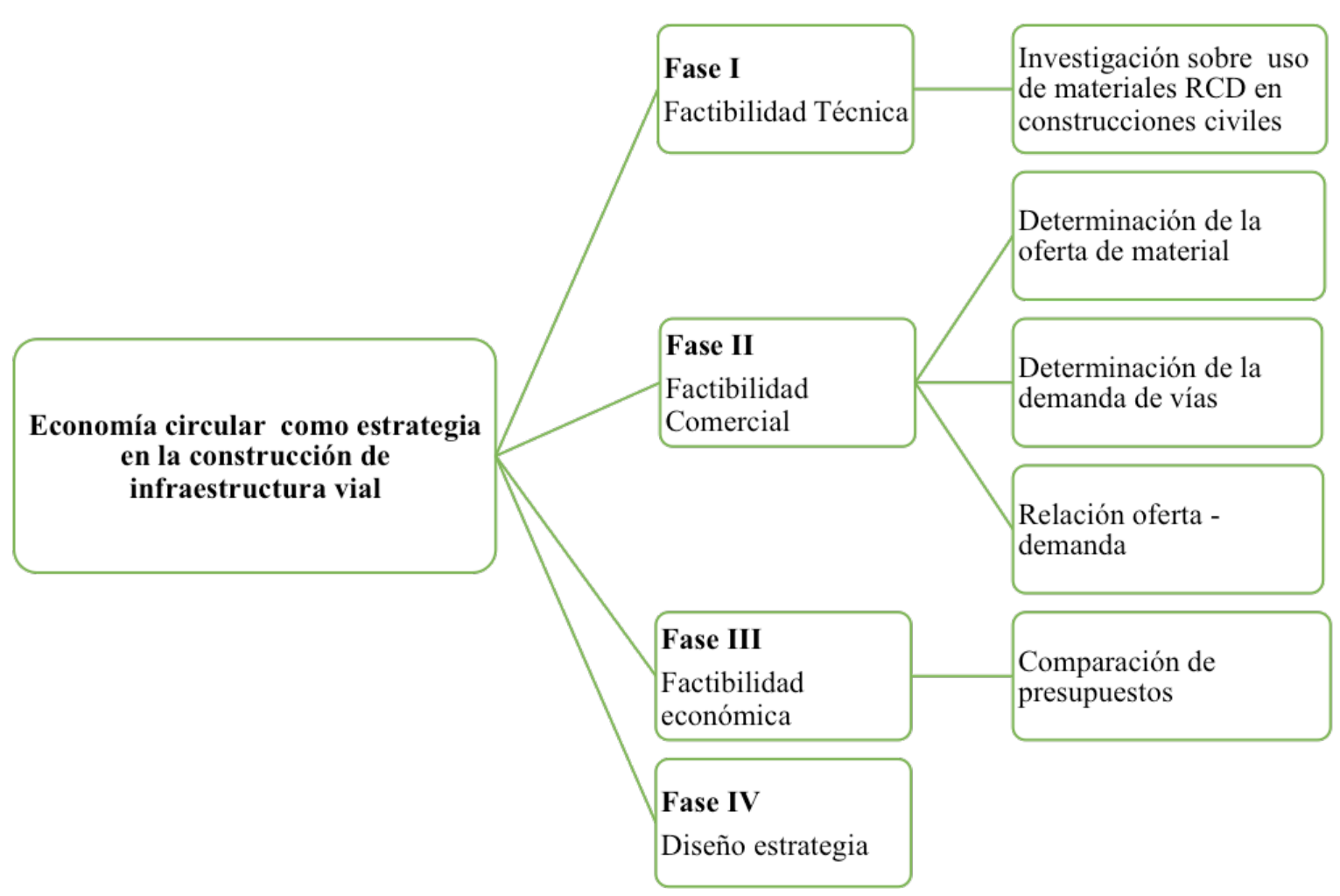

Fuente: elaboración propia.

\section{FASE 1. Análisis de la factibilidad técnica.}

En esta fase se realiza una investigación e inspección de antecedentes relacionados con el uso de los RCD para determinar la participación de dichos materiales en las construcciones civiles, así como la clase de materiales más convenientes para el tipo de construcción de carreteras. Además, se destacan los antecedentes que elaboran ensayos de laboratorio que comprueban las características mecánicas de los materiales en este tipo de construcciones. 


\section{FASE 2. Determinación de la factibilidad comercial.}

Para determinar la factibilidad comercial se tienen en cuenta los siguientes aspectos:

\section{Determinación de la oferta.}

A través de una inspección del sitio, se identifican los proyectos de construcción residencial, los tipos de materiales constructivos a utilizar y el tamaño de los proyectos expresado en áreas. También se estima la cantidad de residuos de construcción generados por los proyectos encontrados.

\section{Determinación de la demanda.}

Las carreteras de segundo y tercer orden se identificaron; aquellas proyectadas para ser construidas en el lugar escogido como estudio de caso. Dicha información es obtenida a través del plan de desarrollo del municipio. Asimismo, se realiza la petición de la información detallada a la entidad pertinente. Además, la Secretaría de Obras Públicas del municipio brindó información acerca de la localización de las vías y de un presupuesto estimado, según la construcción de ellas en placa huella.

Por otro lado, se establece una estructura del pavimento de acuerdo a las recomendaciones del informe de Zonificación Geomecánica de la sabana de Bogotá, información secundaria del municipio de sopó [12]. Así, mediante estudios de suelos, se establecen las recomendaciones de cimentaciones para todo tipo de estructuras.

De igual forma, se determina la cantidad y tipo de material necesario para la construcción de las subbases y bases granulares, según tres (3) propuestas de dimensionamiento de las vías. Se asumen los anchos de calzada de vías secundarias y terciarias de dos (2) carriles, correspondiente a siete (7) y seis (6) metros $(m)$ respectivamente [13], y la propuesta de diseño brindado por la alcaldía de cinco $(5 \mathrm{~m})$ de ancho. 


\section{Relación oferta-demanda.}

Por último, se realiza el análisis de la relación entre la oferta y la demanda del material. Este determina la cantidad de $m$ lineales de carreteras habilitados para construir con el material disponible.

\section{FASE 3. Determinación de la factibilidad económica.}

Para la construcción de un kilómetro de vía, se realiza un comparativo del presupuesto de tres (3) escenarios. En el primero se considera el costo de la construcción de una (1) carretera con materiales convencionales. En el segundo se tiene en cuenta el uso de materiales reciclados y, en el tercero, la construcción de las vías en placa huella ya establecido por la Secretaría de Obras Públicas del municipio.

\section{FASE 4. Planteamiento de estrategia.}

Una estrategia para el uso de residuos de construcción en subbases y bases granulares para vías secundarias y terciarias es aplicada al municipio de Sopó. El tema central es la reutilización de los RC, con el fin de reducir la cantidad de materia prima necesaria y la producción de residuos.

\section{Resultados}

\section{FASE I. Análisis de factibilidad técnica.}

Tras la revisión de la información brindada por los estudios realizados a los RCD, utilizados como material constructivo en las diferentes partes de la estructura de una carretera, se evidencia que las características de estos materiales (como la deformación, módulo resiliente y compactación) son aceptables; su comportamiento mecánico se asemeja al de los agregados naturales utilizados 
convencionalmente. Además, no presentan variaciones considerables que puedan afectar negativamente el comportamiento de los materiales en la estructura, tras ser sometidos a las cargas y condiciones esperadas.

Con base en los informes y resultados revisados, en la construcción de subbase y base de una vía secundaria o terciaria (que presente bajo tráfico vehicular), se recomienda utilizar el 50\% de material granular y 50\% de residuos de construcción; los residuos deben ser de concreto, mortero, agregado fino y agregado grueso. Asimismo, debe estar libre de materiales como la madera, plástico, acero, papel, aceites, entre otros; la presencia de estos puede afectar el comportamiento de los agregados reciclados, al ser sometidos a cargas vehiculares o a la presencia de la humedad.

\section{FASE II. Factibilidad Comercial.}

En cuanto a los resultados obtenidos del estudio realizado de la oferta y la demanda de residuos de construcción, en el municipio de Sopó, para las unidades residenciales se espera alrededor de $7691.66 \mathrm{~m} 3$ de residuos de construcción compuestos por mortero, concreto, agregados finos y agregados gruesos. En este caso se puede disponer del 100\% de los residuos de las construcciones; el municipio se encuentra gestionando una estrategia para la disposición de dichos residuos. Así, los residuos disponibles se podrían disponer de tres (3) formas:

$6.15 \mathrm{~km}$ de vía con las dimensiones establecidas por la alcaldía.

$3.75 \mathrm{~km}$ de vías secundarias.

$4.39 \mathrm{~km}$ de vías terciarias. 
Para la construcción de los seis (6) tramos de vías terciarias, se necesitan 1197 m3 de material para la subbase y 789 m3 para la base granular, para un total de 1995 m3. Adicionalmente, se podría utilizar el material para la ampliación y construcción de 2.5 kilómetros (km) de vías secundarias que requieren 3075 m3 de RC para la subbase y 2050 m3 para la base granular. Con base en lo anterior, se puede determinar que con el material de RC disponible se pueden construir $1.14 \mathrm{~km}$ de vías terciarias y $2.5 \mathrm{~km}$ de vías secundarias.

\section{FASE III. Factibilidad económica.}

\section{Primera propuesta vía con ancho de calzada de 3.8 metros.}

Del presupuesto brindado por el municipio de Sopó, se evidencia, primero, que 1000 m de vía construidos en placa huella cuestan aproximadamente \$572.094.755,15 pesos colombianos (COP), correspondiente a 172715.67 dólares (USD). Segundo, el presupuesto para 1000 m de vía en pavimento flexible, con materiales convencionales, es de aproximadamente \$896.745.528,26 COP (270727.89 USD). Tercero, el presupuesto para 1000 metros de vía en pavimento flexible con RC en las capas granulares es de aproximadamente \$ 788.351.932,84 COP (238003.81 USD).

Debido al bajo costo de construcción en placa huella, el municipio de Sopó ha optado por esta alternativa. Sin embargo, según los presupuestos presentados (con un aumento del 37.8\%) se podría emplear la opción de una vía en pavimento flexible con RC. Esta tendría beneficios como permitir una mayor vida útil de los vehículos que transitan, al tener una superficie plana; reducir el consumo de combustible hasta un $20 \%$ y facilitar el acceso y la movilidad de quienes transitan por estas vías, sin importar el medio de transporte, además de reducir los impactos ambientales utilizando los residuos de construcción. 


\section{Segunda propuesta vía terciaria con ancho de calzada de 6.0 metros.}

El presupuesto para $1000 \mathrm{~m}$ de vía en pavimento flexible con materiales convencionales es de aproximadamente \$1.310.034.524,12 COP (395500.03 USD). El presupuesto para 1000 metros de vía en pavimento flexible con RC en las capas granulares es de aproximadamente \$1.143.273.676,48 COP (345154.85 USD).

Para el caso de la vía terciaría, con las dimensiones recomendadas, se evidencia que el presupuesto disminuye $12.73 \%$. Así, es factible el uso de RC,

económicamente, en la construcción de este tipo de vías.

\section{Tercera propuesta. Vía secundaria con ancho de calzada de 7.0 metros.}

El presupuesto para 1000 metros de vía en pavimento flexible con materiales convencionales es de aproximadamente \$1.558.007.921,63 COP (470363.31 USD). El presupuesto para 1000 metros de vía en pavimento flexible con RC en las capas granulares es de aproximadamente \$1.356.369.449,90 COP (409488.57 USD). De esta manera, con la utilización de RC en las vías se-

cundaria se reduce el costo en un $12.94 \%$, con respecto al costo de la vía construida con materiales convencionales.

\section{FASE IV. Diseño de la estrategía.}

A continuación, se presenta la propuesta metodológica para el uso de RC en subbases y bases granulares en vías secundarias y terciarias. Esto con el fin de ser replicable en cualquier municipio donde se desee estimar y conocer la 
Figura 3. Esquema de la estrategia.

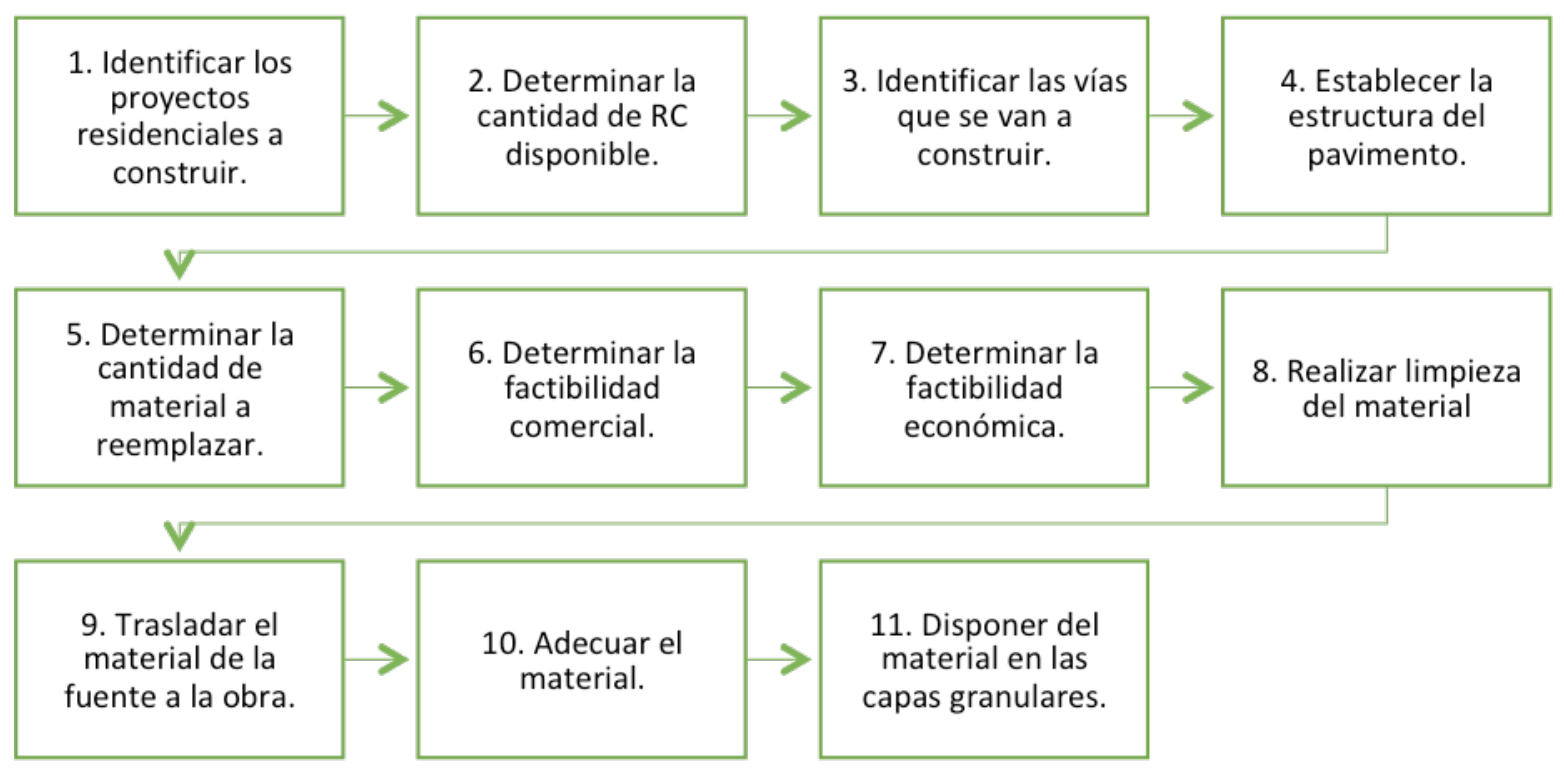

Fuente: elaboración propia.

factibilidad del uso de estos materiales, en reemplazo de los convencionales, según las condiciones locales del lugar donde se quiere replicar la metodología.

\section{Identificar los proyectos residenciales a construir.}

Para determinar los proyectos disponibles en la zona escogida se puede realizar una inspección previa de las licencias de construcción tramitadas para dicha zona. Lo anterior, mediante la base de datos y los informes realizados por

CAMACOL, disponibles para el público. Además, se realiza una visita al lugar y se recorre el sitio para buscar las obras constructivas a realizar en la zona.

\section{Determinar la cantidad de RC disponible.}

Para determinar la cantidad de RC disponible, se realiza una investigación sobre el área total a construir en cada una de las obras seleccionadas. Dicha cantidad, obtenida en $\mathrm{m}^{2}$, se multiplica con el factor de generación, de $0.085 \mathrm{~m} 3$ / 
m2 para el caso de construcciones residenciales. Esto

pues es necesario tener la cantidad de residuos generados en $\mathrm{m}^{3}$, que corresponderá a la oferta de material en la zona.

$$
R C=0.085 \mathrm{~m}^{3} \vee \mathrm{m}^{2} \text { * Área construida }\left(\mathrm{m}^{2}\right)
$$

En efecto, se debe tener en cuenta si la administración del lugar escogido cuenta con una estrategia de disposición de residuos sólidos o escombros. Lo anterior puede disminuir el porcentaje de residuos a disponer para la construcción de las vías seleccionadas.

\section{Identificar las vías que se construirán.}

Las vías que se construirán en el municipio elegido se identifican mediante la revisión del Plan de Ordenamiento Territorial (POT) o del Plan de Desarrollo Municipal, establecido por la administración que esté rigiendo en el periodo de la investigación. Además, se debe realizar la localización de cada

una de las vías a construir para, posteriormente, determinar la distancia ideal de los proyectos constructivos de donde se extraerán los RC para la construcción de las vías. Es recomendado que las vías se encuentren aproximadamente $5 \mathrm{~km}$ de la fuente de material.

\section{Establecer la estructura del pavimento.}

Si ya se cuenta con el diseño de la estructura de pavimentos, se toman los espesores que especificados en el diseño. Si no se tiene el diseño del pavimento, se pueden tomar otros diseños utilizados en la zona como referencia. En caso de no contar con un diseño de pavimento, los espesores se pueden determinar mediante el estudio de suelos de la zona o a través de la revisión de mapas geológicos. Esto, para estimar los espesores de la sub- 
base y base granular, por medio del valor del CBR del suelo donde se construirá la vía. Este tipo de información se puede obtener de los informes de proyectos similares realizados con anterioridad o en la Secretaría de Obras Públicas de cada municipio.

En caso de no tener acceso a la información de estudios de suelos previos o diseño de pavimento, se puede considerar estimar unos espesores según las recomendaciones de cimentación realizadas por INGEOMINAS en los informes de Zonificación Geomecánica.

\section{Determinar la cantidad de material a reemplazar.}

De acuerdo a los espesores de la subbase y base granular, establecidas en el diseño de pavimento, se calcula el volumen total de material necesario

para construir la vía, considerando la longitud total de ésta y el ancho asumido. Además, se debe considerar hasta un 50\% del porcentaje de material a reemplazar, tanto en base como en subbase, para determinar la cantidad necesaria de residuos.

\section{Determinar la factibilidad comercial.}

La determinación de la factibilidad comercial consiste en identificar si la cantidad de RC disponible en la zona es suficiente para la construcción de

las vías seleccionadas. Para esto, la cantidad de material debe ser mayor a la cantidad necesaria para reemplazar el material convencional en la subbase y base de la carretera. De no ser así, y si se desea, se puede establecer la longitud de la vía que se puede cubrir con el material disponible.

\section{Determinar la factibilidad económica.}

La factibilidad económica es determinada a través del presupuesto de la construcción de la vía, utilizando materiales convencionales, y el presupuesto, 
haciendo uso de los materiales reciclados. Para lo anterior es preciso tener en cuenta el transporte del material desde la fuente hasta el lugar de la obra a realizar; la logística o mano de obra que limpiará el material y la trituración del

material en sitio, para conseguir la granulometría establecida por la norma en cada una de las capas granulares. Por último, se comparan los presupuestos y se determina si el uso de los residuos de construcción, como sustituto de los materiales convencionales, es factible económicamente.

\section{Realizar limpieza del material para su uso.}

Los residuos deben estar compuestos por concreto, mortero, agregados finos y agregados gruesos. También se debe verificar la inexistencia de materiales como madera, plástico, metales y/o aceites o sustancias tóxicas dentro del material escogido, previo a trasladar el material de la fuente a la obra.

El traslado del material se realiza en volquetas con una capacidad de 6 m3. Este va desde la fuente (la construcción residencial de donde se obtendrá el material) hasta el sitio donde se realizará la construcción vial. En este sentido, se recomienda que la distancia, entre la fuente y el sitio de disposición, sea de aproximadamente $5 \mathrm{~km}$.

\section{Adecuar el material.}

Una vez se dispone del material en el sitio de la obra, se tritura el material para su uso en la subbase y base de la vía, de acuerdo a las especi-

ficaciones estipuladas por la norma de INVIAS, en los artículos 320 y 330 respectivamente. Igualmente es elemental realizar los ensayos requeridos para verificar que el material cumple con las propiedades necesarias para 
este tipo de estructuras.

\section{Disponer del material en las capas granulares.}

Por último, se dispone del material necesario para la construcción de cada una de las capas granulares de la vía.

\section{Discusión de resultados}

La revisión de la literatura, que compone el resultado de los estudios realizados por diferentes autores alrededor del mundo, muestra la viabilidad del uso de los agregados reciclados en la construcción de carreteras. Esto debido a que las propiedades mecánicas de los materiales utilizados en las secciones de prueba estudiadas cumplen con los requisitos mínimos de calidad según las diferentes normas internacionales.

De acuerdo al análisis realizado en la FASE 1, dichos materiales pueden ser reemplazados hasta en un 50\% (tanto en bases como en subbases). Adicionalmente, otros estudios han revelado los beneficios de utilizar dichos materiales reciclados, como estabilizadores en la subrasante de las vías. Sin embargo, las características de los materiales requieren mayor humedad, inconveniente a la hora de utilizarse en lugares con climas cálidos.

Por un lado, conforme a los resultados y el análisis expuesto en la FASE 2, correspondiente al análisis de la factibilidad comercial, se demuestra la viabilidad en el estudio de caso seleccionado; actualmente se encuentra una fuente de material reciclado de gran tamaño. Igualmente, según la alcaldía municipal, la proyección de las vías a construir permite confirmar la cobertura de la totalidad de la demanda del material con la oferta disponible actualmente. Por otro lado, se encuentra el beneficio económico del uso de los materiales reciclados en las construcciones viales; esta disminuye el costo del km de la vía en comparación con la construcción convencional.

De la mano con los beneficios mencionados anteriormente, se puede destacar el impacto positivo que se puede generar. Con el uso de los ma- 
teriales reciclados, se promueven las alternativas de utilización de residuos de construcción, así como la gestión adecuada de este tipo de materiales, mientras se reduce el consumo del material extraído de fuentes naturales no renovables.

\section{Conclusiones}

Con base en esta investigación se puede concluir que la estrategia realizada es una oportunidad para impactar de manera positiva el desarrollo de vías rurales; reduce el presupuesto de estas obras de forma directa, utilizando los RC a los que posiblemente se les esté dando una mala disposición en la actualidad. Además, contribuye al desarrollo sostenible de las regiones y disminuye notablemente la contaminación y transformación del entrono generada por estos residuos sólidos.

Según la revisión de los antecedes, se evidencia que los residuos de construcción son aptos para usar en la construcción de carreteras, disponiendo de dichos residuos en la estructura granular del pavimento (base y subbase). Lo anterior mantiene las mismas propiedades mecánicas que los materiales convencionales e, incluso, disminuye las deformaciones en las capas superiores de la estructura de pavimento, como en la capa asfáltica.

De acuerdo al análisis de la oferta y la demanda, se demuestra la factibilidad comercial; el material de RC disponible es de 7.691,66 m3 y cubre la demanda de 7.120 m3 de material para su uso en la construcción de seis (6) tramos de vías terciarias que requieren un total de $1.995 \mathrm{~m} 3$ de material reciclado. Por otro lado, para la construcción y/o ampliación de 2,5km de vías secundarias que requieren cerca de $5.125 \mathrm{~m} 3$ de material reciclado.

En cuanto al análisis económico, se evidencia con el uso de los RC en la 
construcción de carreteras se logra una reducción de cerca del 12\% en relación con el costo de la una vía construida con materiales convencionales. Esta reducción se presenta tanto en vías secundarias como en vías terciarias y determina la factibilidad económicamente del uso de los RC.

\section{Referencias bibliográficas:}

[1] Á. Barrios. (2012). Materiales de construcción en construcción sostenible [En línea]. Disponible en: https://www.eoi.es/wiki/index.php/MATERIALES_ DE_CONSTRUCCIÓN_en_Construcción_sostenible

[2] Ministerio de Ambiente y Desarrollo sostenible. (2017), "Disposición final de RCD” [En línea]. Disponible en: https://www.cornare.gov.co/memorias/ memorias-rcd/Ministerio-de-Ambiente-y-Desarrollo-Sostenible.pdf

[3] G. Ospina, "El papel de las vías secundarias y los caminos vecinales en el desarrollo de Colombia", Revista de Ingeniería, vol. O(44), no. 20, pp. 20-27, 2016. [En línea]. Disponible en: https://doi. org/10.16924/riua.v0i44.911

[4] Ministerio de Ambiente y Desarrollo Sostenible. (2018). Colombia le apuesta a las 9R en Economía circular [En línea]. Disponible en: http://www. minambiente.gov.co/index.php/noticias/4225-colombia-le-apuesta-a-las-9r-en-economia-circular?fbclid=IwAROpLosRWi9c64HoytYAROKYsvT32vtr5WDnbvNnaJQXMptpIdQCInS1 wyO

[5] Decreto 1115/2012, de 7 de septiembre, por medio de la cual se adoptan los lineamientos Técnico-Ambientales para las actividades de aprovecha- miento y tratamiento de los residuos de construcción y demolición en el Distrito Capital.

[6] Decreto 1017/2015, de 26 de mayo, por medio del cual se expide el Decreto Único Reglamentario del Sector Trabajo.

[7] Decreto 2981/2013, de 20 de diciembre, por el cual se reglamenta la presentación del servicio público de aseo.

[8] A. Ortega, H. Casas y Y. Figueroa, Guía Para La Elaboración Del Plan De Gestión De Residuos De Construcción Y Demolición - Rcd En La Obra. Bogotá: Secretaria Distrital de Ambiente, 2015 [En línea]. Disponible en: http://www.ambientebogota.gov.co/ web/publicaciones-sda/cartilla-rcd

[9] J. Cárdenas, Diseño Geométrico de Carreteras (2da Ed). Bogotá: ECOE Ed., 2014.

[10] INVÍAS, "Capítulo 3. Afirmados, subbases y bases" en Normas y especificaciones, Bogotá: INVIAS, 2012, pp. 1-150.

[11] M. Agudelo y J.P. Rodríguez, "Estimación de generación y composición de residuos de construcción en la ciudad de Villavicencio", en $2014 \mathrm{~V}$ Congreso Internacional de Ing. Civil, pp. 1-11. 2014

[12] Y. Calderón y A. Ruiz, Proyecto Compilación y Levantamiento de la Información Geomecánica. Zonificación Geomecánica de la Sabana de Bogotá: Hidrología y clima. Volúmenes IV a VI. Bogotá: INGEOMINAS, 2004.

[13] Construdata, "Componentes de las vías", 2012. [En línea]. Disponible en: http://www.construdata.com/Bc/Revista_Construdata/Articulos/ componentes_de_las_vias.asp?fbclid=IwAR2g-pht8Xe5m2eNC9hOv2RwwgdRgKWd5j9Xxsx_kfqIRBjmlbwK4m_k93M 